\title{
A New Extra-Abdominal Channel Alternative to the Mitrofanoff Principle: Experimental and Preliminary Clinical Experience
}

\author{
Antonio Macedo Jr., Tiago Rosito, Jesus A. S. Pires, Riberto Liguori, Valdemar Ortiz \\ Department of Urology, Federal University of Sao Paulo, Unifesp, Sao Paulo, SP, Brazil
}

\begin{abstract}
Introduction: The appendix is the gold-standard channel for the Mitrofanoff principle in pediatric urology, but the search for alternatives is justified considering it may not be available or preferably used for colonic stomas (Malone antegrade continence enema). The aim of this study is to report on technical feasibility of a new approach for creating catheterizable channels in a rabbit model and to present our preliminary clinical experience.

Material and Methods: We configured a tube from two rectangular skin flaps 1x4 cm opposite each other in the middle line of the lower inferior abdomen. The channel was anastomosed to the bladder dome with embedding sutures to create a valvular mechanism. The experimental study consisted of 12 rabbits, divided in 4 groups according to the sacrifice schedule at 2, 4, 8 and 12 weeks. At $30^{\text {th }}$ postoperative day, an urodynamic evaluation was performed to record continence of the stoma. A histological analysis of the specimens stained with hematoxylin-eosin, Masson trichrome and Picrosirius red was also done in group 2 (sacrifice at 4 weeks postoperatively). We used this method in 3 patients with congenital non-neurogenic bladder disease presenting with massive residual volumes without compliance deficits.

Result: The technique proved feasible in all animals, 9 of 12 could be easily catheterized and underwent urodynamic study. No stoma leakage was observed in 7 animals at high bladder pressures $\left(>50 \mathrm{~cm} \mathrm{H}_{2} 0\right)$ and only 2 animals had some leakage at $40 \mathrm{~cm} \mathrm{H}_{2} 0$. Urodynamics performed through the stoma showed urethral leakage at $20 \mathrm{~cm} \mathrm{H}_{2} 0$, therefore demonstrating the efficacy of the valvular mechanism. Histological analysis confirmed good integration between the tube and the bladder. Mean follow-up of the clinical series (3 patients) was 7.2 months. Two patients remained continent up to 4 hours, whereas 1 patient had some leakage after 2 hours.

Conclusion: We were able to confirm feasibility of a new extra-abdominal channel based on the Mitrofanoff principle and successfully reproduced the method in a clinical setting. Follow-up was short and long term results are required before any conclusive judgment can be made.
\end{abstract}

Key words: bladder; children; urinary diversion; Mitrofanoff principle; surgery

Int Braz J Urol. 2009; 35: 205-16

\section{INTRODUCTION}

The introduction of clean intermittent catheterization (CIC) at the 70s improved considerably the quality of life in patients with neurogenic bladder and other end-stage bladder disease (1). The appendicovesicostomy gave patients more comfort and autonomy, especially for those confined to a wheel-chair or boys with urethral sensitivity (2). The appendix is the gold-standard channel for urinary reservoirs but simultaneous need for a MACE (Malone antegrade continence enema) procedure and urinary reconstruction forced urologists to find alternatives to the appendix as the outlet tube. The Yang-Monti, double Monti and Casale tubes are good options but they still have complications and are constructed from 
intestinal segments, which could be avoided when there is no need for bladder augmentation but only a Mitrofanoff channel in the native bladder (3).

A second point of concern in urinary and colonic continent stomas is the high incidence of stoma stricture, which might be related to variables like the technique itself, frequency of catheterization and presence of feces, although this latter condition has not yet been proved $(4,5)$. To date, a revision rate of $10-50 \%$ due to stoma stricture has been reported in the literature.

The aim of this study was to report on technical feasibility of a new approach for creating catheterizable channels in a rabbit model and to present preliminary clinical results. The technique was named RPM because they are the initials of three authors who developed the concept (Rosito, Pires and Macedo). One possible advantage of this method is in cases where it is necessary to create an abdominal channel for catheterizing the native bladder without need of opening the peritoneum to obtain the appendix or make a Yang-Monti tube thus reducing considerably the morbidity of the treatment.

\section{MATERIALS AND METHODS}

We selected the rabbit for this experimental model because of its practical features, including ease of manipulation and familiarity of our group with this model in previous experimental studies. The experimental protocol was reviewed and approved by the Local Animal Research Committee. A total of $12 \mathrm{New}$ Zealand White Rabbits, approximately eight weeks old and a weight of 2.5-3.0 kg were acclimated at the Experimental Research Animal Surgery Department for one week before the procedures.

The rabbits were anesthetized intramuscularly with ketamine hydrochloride $(30 \mathrm{mg} / \mathrm{kg})$ and xylazine $(5 \mathrm{mg} / \mathrm{kg})$, and local anesthetic (xylocaine) was used to perform a penile block. All animals were operated on under sterile conditions and under optical magnification (2.5X). We made two rectangular flaps $(1 \times 4 \mathrm{~cm})$ both opposite each other in the middle line of the lower inferior abdomen (Figure-1A). The vascular structure of both flaps was kept intact by inferior superficial epigastric vessels and superficial iliac circumflex.
The cranial and lateral surface of the flaps was sectioned, giving it enough mobility to allow a 90degree rotation. The horizontal superior border was moved to the vertical position close to each other. A 5.0 polyglicolic acid running suture was performed configuring a skin plate (Figure-1B). The next step consisted of an anastomosis of the lateral margins of the flaps using a $10 \mathrm{~F}$ plastic tube as a mold in order to create a tube (Figure-1C).

A small abdominal incision to reach the bladder was performed and a $0.5 \mathrm{~cm}$ section of the anterior wall at the dome level of the bladder was performed (Figure-1D). The proximal end of the tube was anastomosed to the bladder by means of 6-8 5.0 polyglicolic acid sutures (Figures $2 \mathrm{~A}$ and $\mathrm{B}$ ). The continence mechanism of the channel was done by embedding it over 3, 4.0 poliglycolic acid sutures at the seromuscular wall of the bladder. The abdominal wall was closed in layers and the stoma consisted of the distal end of the tube, which was adapted to the wound margins without circular anastomosis (Figure-2C). The animals were kept in a warm room with ventilatory support until they were well awake. The channel mold was left intact for 7 days. The experimental study consisted of 12 rabbits, divided in 4 groups according to the sacrifice schedule at 2, 4, 8 and 12 weeks (groups 1 to 4 respectively).

We evaluated patency of the stoma and performed urodynamic analysis at sacrifice (group 1) or 30 th postoperative day when animals were sedated with midazolam $0.02 \mathrm{mg} / \mathrm{kg} \mathrm{IM}$ and a $10 \mathrm{~F}$ plastic tube insertion was attempted (Figure-2D). At this moment, an urodynamic evaluation was completed using a Dynamed set, (Sao Paulo, Brazil). We catheterized the bladder initially through the urethra using a $4 \mathrm{~F}$ catheter for filling the bladder and a second one via the stoma for recording bladder pressure. A rectal catheter with a balloon was used to record abdominal pressure. We changed the filling/recording function of catheters for subsequently evaluating continence through the urethra and stoma. In order to better define detrusor leak-point pressure (DLPP) through the stoma we performed manual compression of the urethra to avoid overflow through the urethra and created a "stress" study for the channel continence mechanism. Detailed results are shown in Table- 1 and were compared statistically using a Chi-square analysis. Animals of group 


\section{Channel Alternative to the Mitrofanoff Principle}
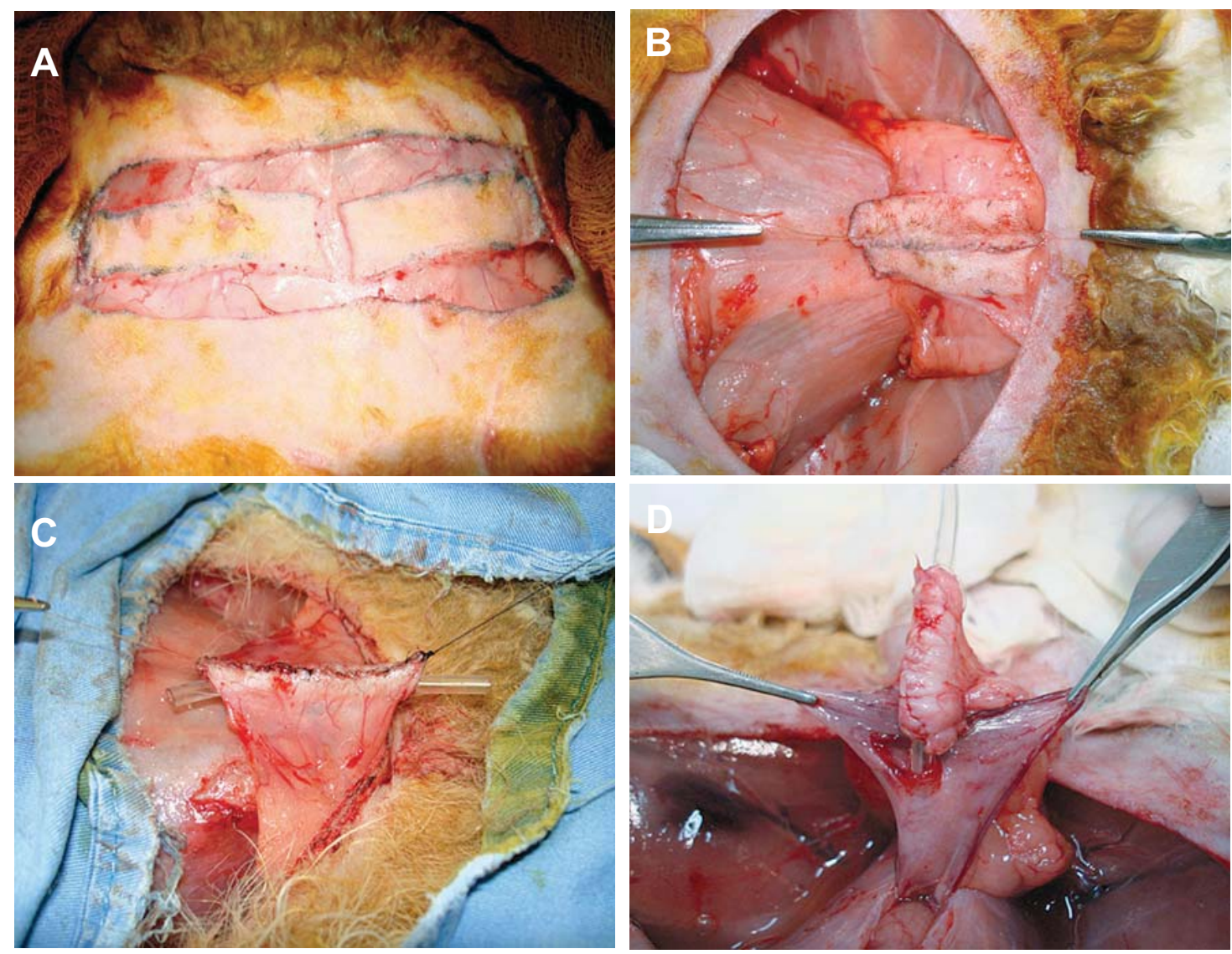

Figure 1 - Two rectangular skin flaps $1 \times 4 \mathrm{~cm}$ opposite each other in the middle line of the lower inferior abdomen are anastomosed as an "onlay" to create a tube to be connected to the bladder dome.

2 were sacrificed and surgical specimens removed, fixed in formalin and sent for histological evaluation stained with hematoxylin-eosin, Masson trichrome and Picrosirius red.

After initial experience with the experimental model, we designed a clinical protocol and informed parents about potential advantages of the technique, mainly to avoid opening the peritoneal space and possible complications were also mentioned. The protocol was also approved by local Ethics Committee. We then introduced the method in clinical practice. We operated on three children presenting with non-neurogenic congenital bladder abnormalities presenting with massive residual volumes without storage deficiency. One patient had presumably primary bladder neck obstruction (Figures 3 and 4), one had posterior urethral valve and a third boy had prune-belly syndrome (Figure-5). Age at surgery and additional surgical procedures are described in Table-2. A stoma catheter (12F silicone Tube) was left indwelling for three weeks when a nurse-urotherapist trained patients how to perform CIC. Patients were followed as outpatients every month for at least 5 months for continence, urinary tract infection occurrence and stoma complications.

\section{RESULTS}

Experimental: There were minor complications related to the operative procedure in 2 cases: wound infection and partial wound dehiscence. At the sacrifice schedule for group 1 and 30th postoperative day for other groups, animals were examined as re- 


\section{Channel Alternative to the Mitrofanoff Principle}
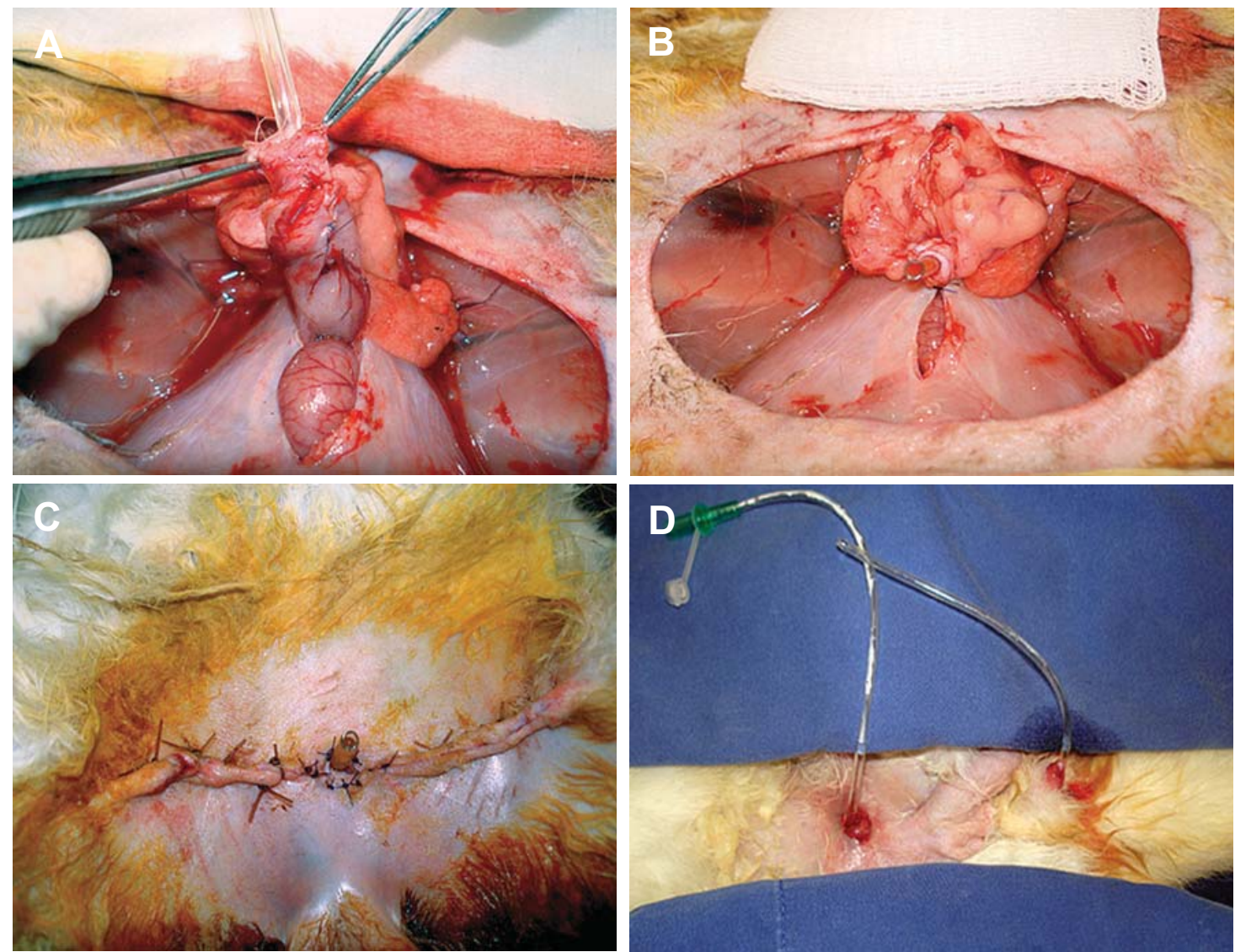

Figure 2 - Anastomosis of the tube in the bladder and final aspect of the stoma. Notice that a catheter is inserted through the stoma and exits through the urethra proving patency of the channel.

Table 1 - Urodynamic records of postoperative evaluation at 30th postoperative day (except animals group 1).

\begin{tabular}{cccccc}
\hline Animal & $\begin{array}{c}\text { UD per Urethra } \\
\text { DLPP } \\
\left(\mathrm{cm} \mathrm{H}_{2} 0\right)\end{array}$ & $\begin{array}{c}\text { Maximal } \\
\text { Pressure }\left(\mathrm{cm} \mathrm{H}_{2} 0\right)\end{array}$ & $\begin{array}{c}\text { Cistometry } \\
(\mathrm{mL})\end{array}$ & $\begin{array}{c}\text { UD per stoma } \\
\text { DLPP } \\
\left(\mathrm{cm} \mathrm{H}_{2} 0\right)\end{array}$ & $\begin{array}{c}\text { Cistometry } \\
(\mathrm{mL})\end{array}$ \\
\hline 1 & 40 & 50 & 80 & 20 & 30 \\
2 & 40 & 60 & 70 & 20 & 20 \\
3 & No loss & 110 & 100 & 20 & 30 \\
4 & No loss & 100 & 70 & 20 & 30 \\
5 & No loss & 65 & 100 & 20 & 25 \\
6 & No loss & 100 & 80 & 15 & 20 \\
7 & No loss & 60 & 80 & 15 & 30 \\
8 & No loss & 60 & 100 & 20 & 25 \\
9 & No loss & 60 & 80 & 20 & 25 \\
\hline
\end{tabular}

$D L P P=$ detrusor leak-point pressure. 


\section{Channel Alternative to the Mitrofanoff Principle}

gards the ease of catheterization, which was possible in 9 of 12 animals. The three failed cases included one animal with acute stricture of the stoma due to intense inflammatory response and the two others that developed wound dehiscence due to local infection.

Urodynamic evaluation was performed through both urethral and abdominal access at the same time. The detailed data are presented in Table-1. In summary, we found no leakage through the stoma in 7 of 9 animals reaching a detrusor pressure ranging from 60 to $110 \mathrm{~cm}$ $\mathrm{H}_{2} 0$. The two other animals had leakage at $40 \mathrm{~cm} \mathrm{H}_{2} 0$. Maximal cystometric capacity ranged from 70 to $100 \mathrm{~mL}$. In order to better evaluate the resistance pressure of the valve mechanism we had to perform manual compression of the urethra because urodynamics performed through a stoma catheter showed leakage at $15-20 \mathrm{~cm} \mathrm{H}_{2} 0$ through the urethra and no leakage at all through the stoma. These figures proved efficacy of the valvular mechanism of the tube when compared mean DLPP (or pressure at maximal capacity when no leakage occurred) in both situations ( $\mathrm{p}$ $<0.05)$. An illustration of the urodynamic curve is shown in Figure-6.

The histological analysis of the specimen showed good integration of the skin tube with the bladder, in some areas with loss of epithelium however without ulceration (Figure-7).

Clinical: To date, our clinical experience of 3 patients presents good results with a mean follow-up of 7.2 months. Two patients manage to catheterize their bladders through their stoma 4-5 times a day without urinary leakage at 4 hour intervals. One patient (case 1) complained of painful catheterization and urinary loss after 2 hours interval between CIC after 5 months of uneventful outcome. In two cases,
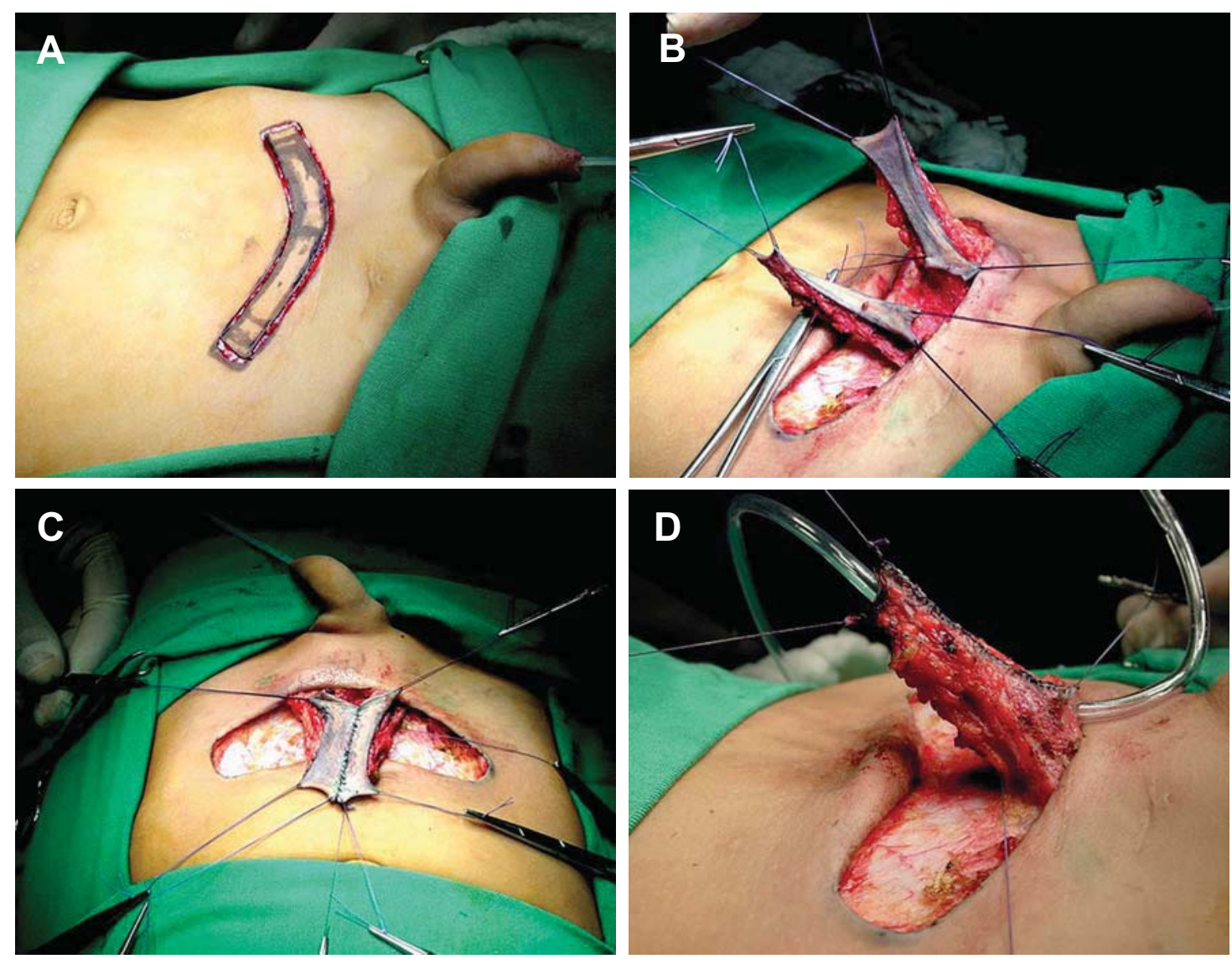

Figure 3 -Construction of the tube with two skin flaps in the first patient operated with this technique. 


\section{Channel Alternative to the Mitrofanoff Principle}
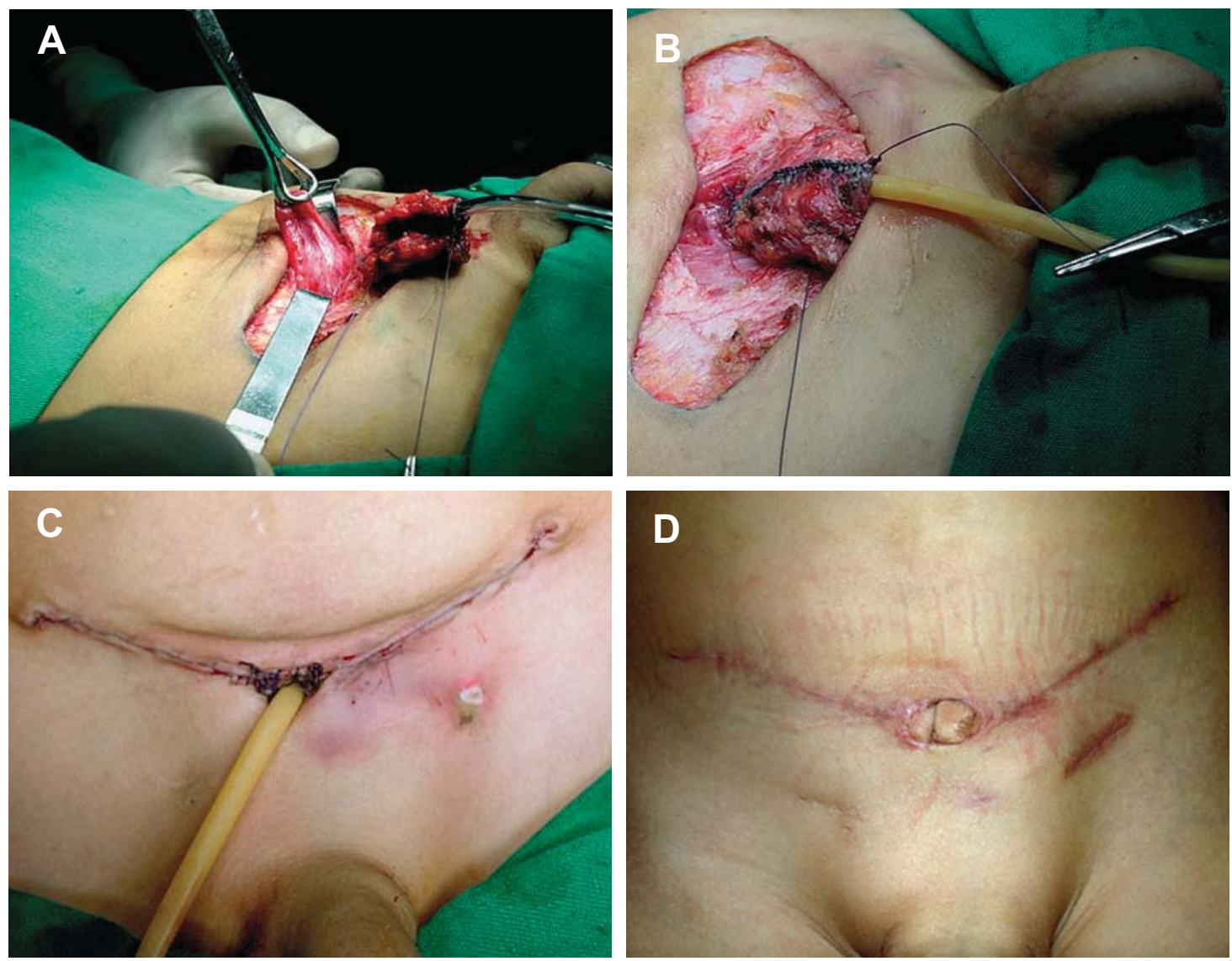

Figure 4-Anastomosis of the tube to the bladder dome and final aspect of the stoma at 60th postoperative day (Figure-4D).

the RPM channel was performed with association of other procedures: a Cohen reflux repair and a Monfort abdominoplasty with orchiopexy.

\section{COMMENTS}

Continent urinary diversion requires an outlet that maintains continence but allows easy catheterization for voiding. Although none of the available options is ideal, the appendiceal flap-valve channel first described by Mitrofanoff appears the most reliable (1). Mitrofanoff principle is a well-established procedure in pediatric urology and main complications reported in the literature with this method are stoma stricture $(8-39 \%)$, leakage (5-22\%) and less frequently appendix necrosis and prolapse (3). In cases where the appendix is unavailable, ileum has been shown to be a suitable alternative. Although some studies have reported higher stoma complication rates with ileal catheterizable conduits, other studies have shown favorable results $(2,3)$.

The Yang-Monti tubes, as well as their modifications, are the best alternative today specially for obese patients, however complications are even higher than the classical appendicovesicostomy and they require open access to the abdomen. This may not be a problem when bladder augmentation is also performed, but when the main problem is abdominal access for CIC the search for a better option is still justifiable.

The ureter has also been used to construct a Mitrofanoff channel. However, in the studies of Van Savage et al. (4) there was a higher risk of complica- 


\section{Channel Alternative to the Mitrofanoff Principle}

tions due to the need for associated ureteral reconstruction (reimplantation or transureteroureterostomy) as well as a greater risk of stoma stenosis.

The technique here presented based on two lower abdominal skin flaps (RPM) could also be regarded as a valuable alternative for continent urinary diversion, mainly because it is an extra peritoneal approach precluding intestinal opening and anastomosis, which theoretically could reduce clinical morbidity. A second potential advantage is the lower risk for stoma stricture, since the two flap anastomosis produce an "onlay" tube without circular anastomosis. If this hypothesis proves to be correct with long term follow-up this technique may gain acceptance especially because Thomas et al. (5) recently reported that up to $50 \%$ of stoma strictures are treated surgically.
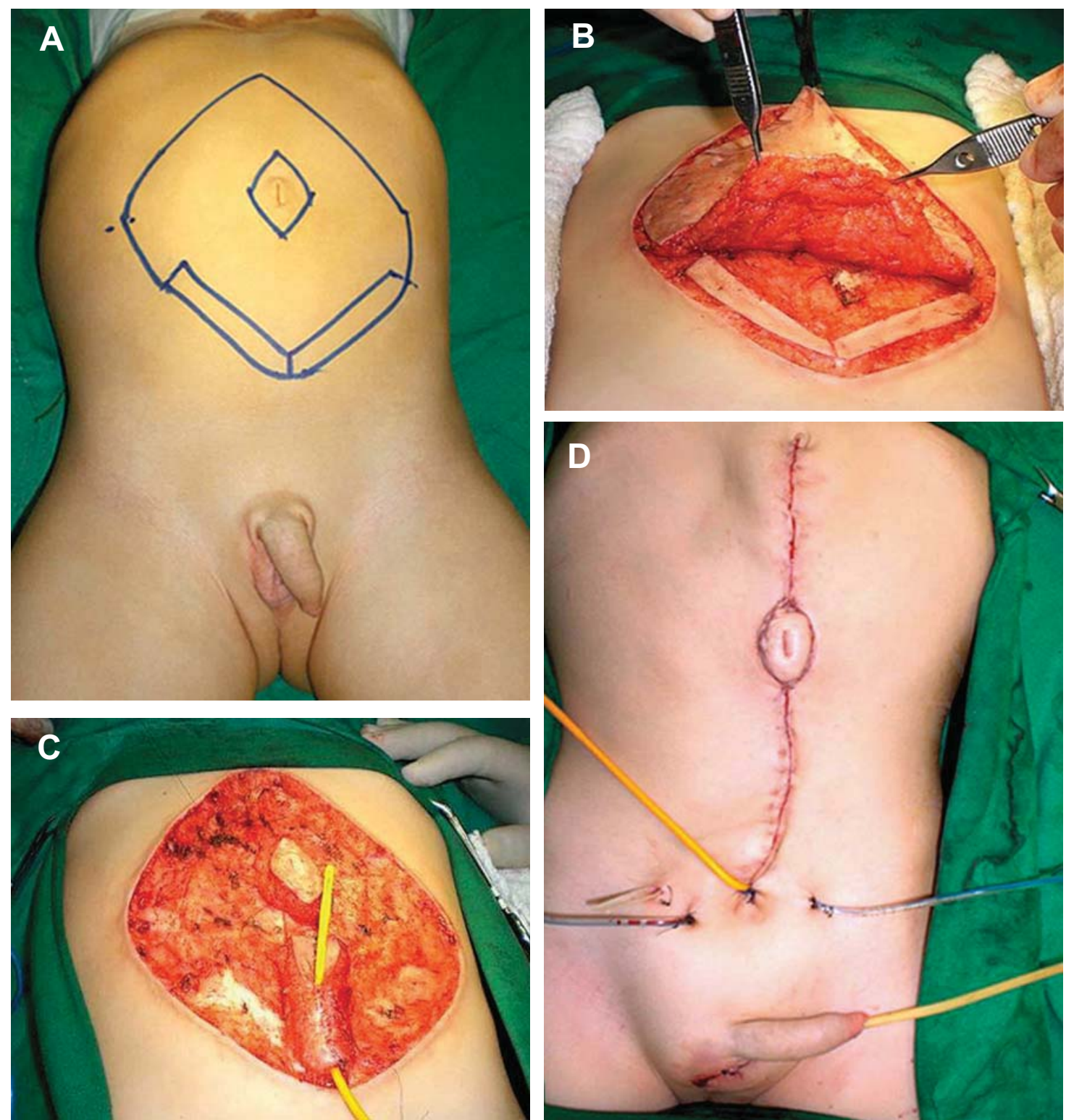

Figure 5 - The technique associated to a Monfort abdominoplasty in one patient with prune-belly syndrome. 
Table 2-Clinical status of patients.

\begin{tabular}{|c|c|c|c|c|c|}
\hline Case & $\begin{array}{c}\text { Age } \\
\text { (years) }\end{array}$ & Diagnosis & $\begin{array}{l}\text { Follow-up in } \\
\text { Months }\end{array}$ & Additional Procedure & Present Status \\
\hline 1 & 4 & $\begin{array}{c}\text { Primary bladder neck } \\
\text { obstruction }\end{array}$ & 10 & none & $\begin{array}{c}\text { Painful catheterization, } \\
\text { leakage after } 2 \text { hours }\end{array}$ \\
\hline 2 & 6 & PUV & 7 & Cohen reflux repair & Continent $>4$ hours \\
\hline 3 & 2 & Prune-belly syndrome & 5 & $\begin{array}{l}\text { Monfort abdominoplasty } \\
\text { and bilateral orchiopexy }\end{array}$ & Continent $>4$ hours \\
\hline
\end{tabular}

The inspiration for a two flap anastomosis to obtain a tube and not simply tubularizing one flap originated from the background of hypospadias repair that suggests that onlay repair is superior to one circular suture in terms of stricture rate. On the other hand, we also learned that these strictures may eventually occur with time and at the present moment we can not predict future of the tube described as a channel. During the peer-review process of this paper, one of the consultants mentioned a video available at the "you tube: http:// www.youtube.com/ watch? $\mathrm{v}=56 \mathrm{wNX} 4 \mathrm{WKSro"}$ " with a similar but different concept of creating a neo-urachus by tubularizing a vertical flap of skin to communicate the bladder with the umbilicus. To our knowledge this procedure has not yet been reported in the literature and therefore we cannot comment on results or ethical aspects. It also differs from our technique as a circular anastomosis is used and theoretically more prone to stenosis or other complications.

We acknowledge that there other limitations of our study. The presence of hair in abdominal skin, mainly in males, could be a possible factor for producing stones. On the other hand, different from the urethra, urine will not be in permanent contact with the luminal surface of the tube, so we can not predict its evolution. We agree that the tube, if continent, might have a drop of urine deposited along the channel but mainly it is presumably only a conduit for CIC, and the role of skin inside the tube is not predictable. Some new methods of hair deepitelization with laser before surgery may also in the future prevent this complication, although there are not objective data currently available, to our knowledge, to support this procedure or its use only in selected cases after complications due to hair inside the channel.

We also acknowledge that vascular support of our channel, which is different from the appendix or Monti procedure originates from superficial vessels in the skin (epigastric and circumflex branches) so that caution should be observed in reoperations, although it would not limit any major abdominal operation when incision is performed above the stoma.

Our experimental data, in which an indwelling catheter in the channel could be left for only 7 days resulted in easy catheterization in $75 \%$ of cases (9 of 12). Histological evaluation confirmed good integration between the skin channel and bladder. Urodynamic evaluation confirmed efficacy of the valvular mechanism. The embedding sutures of the bladder over the channel created at least a $40 \mathrm{~cm} \mathrm{H}_{2} 0$ pressure resistance whereas urethral resistance recorded in the study was $20 \mathrm{~cm} \mathrm{H}_{2} 0$. Our group recently published an experimental ex-vivo model confirming efficacy of embedding sutures in creating resistance also in intestinal reservoirs and intestinal segments (6). We also applied this method for creating channel resistance in our concept of bladder augmentation or substitution over the past ten years (7). Our clinical series confirmed easy CIC in the three patients and continence with a mean follow-up of 7.2 months. Only one patient complained of pain during catheterization after 8 months with no abnormalities.

A third intuitive advantage of the method is the absence of wound skin anastomosis to the tube, since the tube is only adapted to the wound. We hy- 
pothesized a possible impairment of stoma stenosis with our method. Overall, published stoma stenosis rates vary from $3 \%$ to $61 \%(8-10)$. Liard et al. (11) have the longest follow-up of 20 years and had stoma stenosis rates of up to $61 \%$, compared with Horowitz et al. (12) who only had a 3\% stoma stenosis rate, however, with only a short follow-up. Use of the cutaneous anastomosis technique with the incorporation of U-, V- or VZ-flap may also reduce stoma stenosis $(13,14)$, however our method precludes any of these procedures and aesthetic aspect is also very favorable as seen in Figures 4D and 5D.
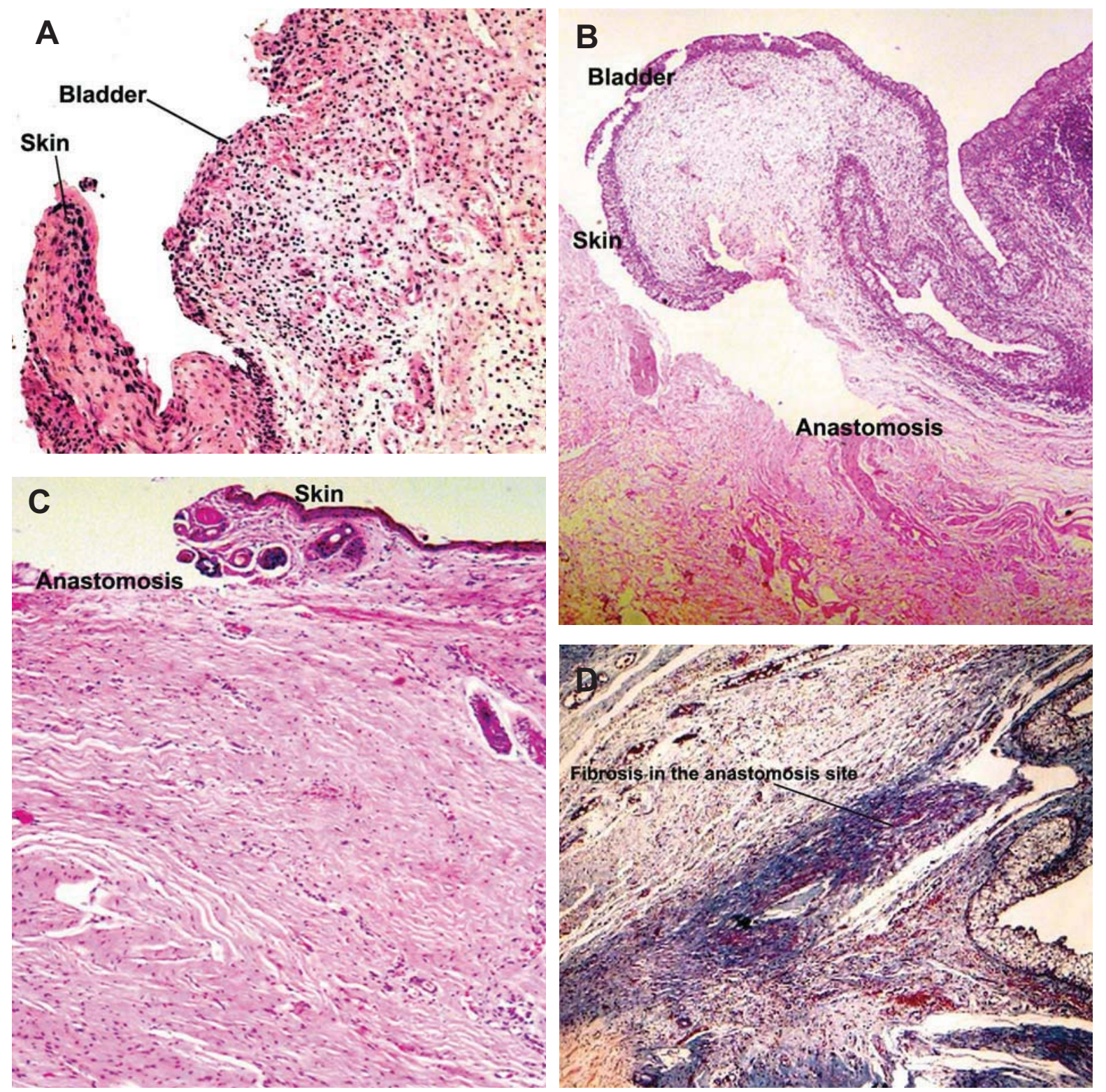

Figure 6-Histological analysis of the specimen showed good integration of the skin tube with the bladder, in some areas with loss of epithelium however without ulceration. 


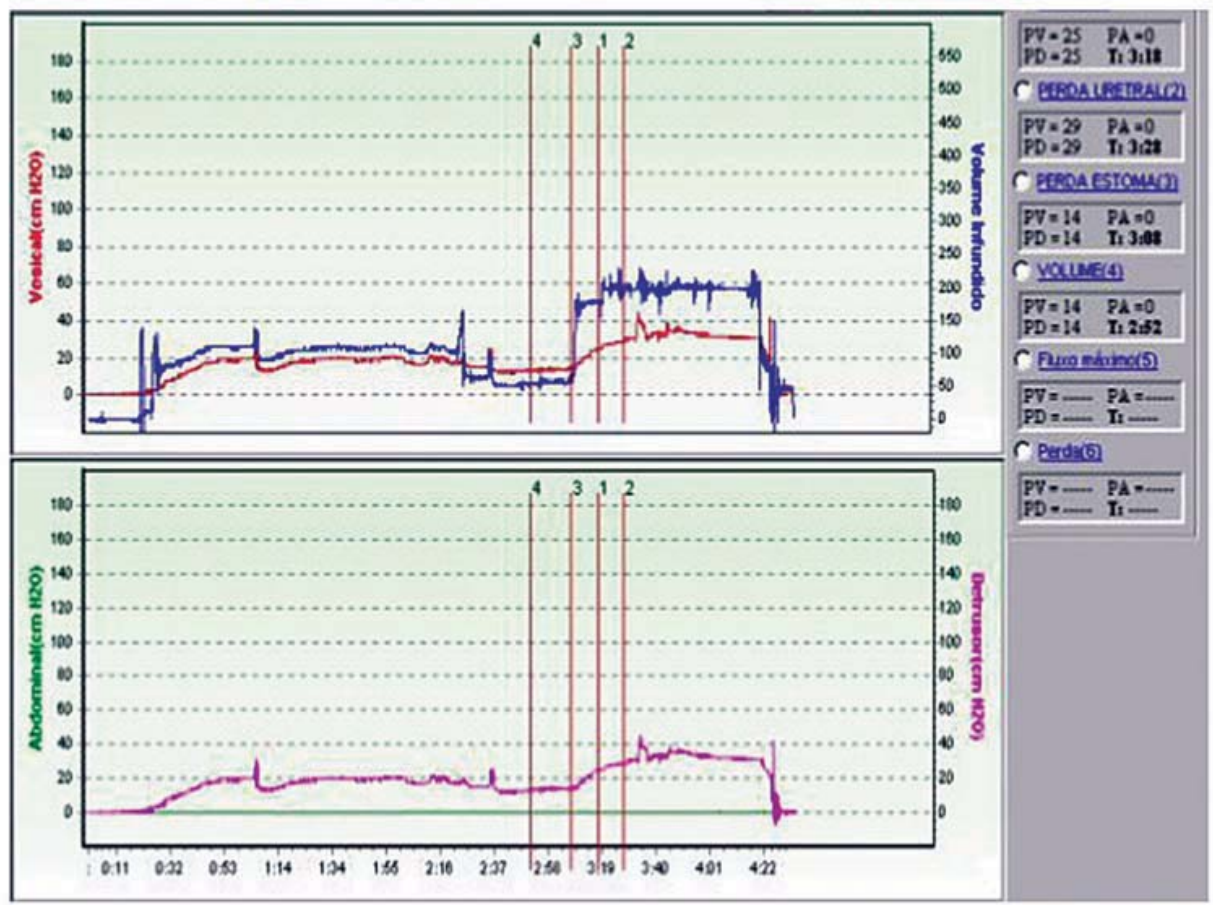

Figure 7 - Urodynamic evaluation of detrusor leak-point pressure through the urethra ( $20 \mathrm{~cm} \mathrm{H}_{2} \mathrm{O}$ : at first half of the examination) and

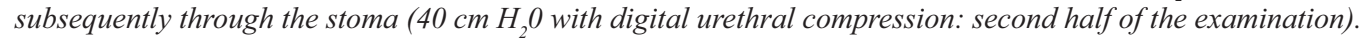

In conclusion, we report our experimental and preliminary experience with a new approach for extra-abdominal channel construction based on the Mitrofanoff principle. The main advantages of our approach are the easy of the technique, applying well-known principles of onlay skin flap anastomosis like in hypospadia repair, minimal invasive access (extra-peritoneal) to the bladder and no need of skin flap anastomosis to the channel (possible impairment of stoma strictures). Long term results are definitely required before any conclusive judgment but preliminary results are very favorable and technical feasibility of the method could be proved in the experimental study.

\section{CONFLICT OF INTEREST}

None declared.

\section{REFERENCES}

1. Mitrofanoff P: Trans-appendicular continent cystostomy in the management of the neurogenic bladder. Chir Pediatr. 1980; 21: 297-305.

2. Cain MP, Casale AJ, King SJ, Rink RC: Appendicovesicostomy and newer alternatives for the Mitrofanoff procedure: results in the last 100 patients at Riley Children's Hospital. J Urol. 1999; 162: 1749-52.

3. Narayanaswamy B, Wilcox DT, Cuckow PM, Duffy PG, Ransley PG: The Yang-Monti ileovesicostomy: a problematic channel? BJU Int. 2001; 87: 861-5.

4. Van Savage JG, Khoury AE, McLorie GA, Churchill BM: Outcome analysis of Mitrofanoff principle applications using appendix and ureter to umbilical and lower quadrant stomal sites. J Urol. 1996; 156: 1794-7.

5. Thomas JC, Dietrich MS, Trusler L, DeMarco RT, Pope JC 4th, Brock JW 3rd, et al.: Continent catheterizable channels and the timing of their complications. J Urol. 2006; 176: 1816-20; discussion 1820. 
6. Vilela ML, Furtado GS, Koh I, Poli-Figueiredo LF, Ortiz V, Srougi M, et al.: What is important for continent catheterizable stomas: angulations or extension? Int Braz J Urol. 2007; 33: 254-61; discussion 261-3.

7. Macedo A Jr, Srougi M: A continent catheterizable ileum-based reservoir. BJU Int. 2000; 85: 160-2.

8. Süzer O, Vates TS, Freedman AL, Smith CA, Gonzalez $\mathrm{R}$ : Results of the Mitrofanoff procedure in urinary tract reconstruction in children. Br J Urol. 1997; 79: 279-82.

9. McAndrew HF, Malone PS: Continent catheterizable conduits: which stoma, which conduit and which reservoir? BJU Int. 2002; 89: 86-9.

10. Cain MP, Rink RC, Yerkes EB, Kaefer M, Casale AJ: Long-term follow-up and outcome of continent catheterizable vesicocstomy using the Rink modification. J Urol. 2002; 168: 2583-5.

\section{Correspondence address:}

Dr. Antonio Macedo Jr.

Rua Maestro Cardim, 560/215

São Paulo, SP, 01323-000, Brazil

Fax: + $55113287-0639$

E-mail: macedo.dcir@epm.br
11. Liard A, Séguier-Lipszyc E, Mathiot A, Mitrofanoff P: The Mitrofanoff procedure: 20 years later. J Urol. 2001; 165: 2394-8.

12. Horowitz M, Kuhr CS, Mitchell ME: The Mitrofanoff catheterizable channel: patient acceptance. J Urol. 1995; 153: 771-2.

13. Kaefer M, Retik AB: The Mitrofanoff principle in continent urinary reconstruction. Urol Clin North Am. 1997; 24: 795-811.

14. Clark T, Pope JC 4th, Adams C, Wells N, Brock JW 3rd: Factors that influence outcomes of the Mitrofanoff and Malone antegrade continence enema reconstructive procedures in children. J Urol. 2002; 168: 153740; discussion 1540.
Accepted after revision: January 20, 2009

\section{EDITORIAL COMMENT}

The article by Macedo et al. in this issue of the International Braz J Urol provides us with an interesting addition to the surgical armamentarium for fashioning continent catheterizable abdominal channels, further expanding the options that followed Mitrofanoff's groundbreaking contribution. This work provides exciting data on animal experiments that translated into therapeutic interventions in a small, selected and somewhat heterogeneous group of children followed-up for a relatively short period of time. Acknowledged as a preliminary experience, the data needs to mature prior to declaring it equivalent or superior to current techniques. The widespread accep- tance of appendicovesicostomy and bowel-fashioned conduits will now face the challenge of options such as the RPM (Rosito, Pires and Macedo) technique, Perovic's genital skin flap (1) or the continent vesicocutaneous channel by Rackley et al. (video quoted in the Discussion section of the article); all attractive as they appear potentially easier to perform but characterized by a different risk/benefit profile. By virtue of avoiding the use of bowel, problems such as internal hernias, anastomotic leaks, mucous production and intra-peritoneal adhesions may be avoided. The trade-off will likely be a different set of complications particularly related to the use of skin flaps for 
intermittent access to bladder drainage. For example, also borrowing from the experience with hypospadias repair, there are specific potential problems that may be of clinical relevance, such as those related to the development of hair follicles within the conduit following puberty. Ultimately, comparative analyses will be needed in order to determine if important long-term outcomes such as stomal stenosis, strictures, leakage and difficulty catheterizing favor one technique over the other. Only time will tell if skin proves to be a suitable alternative to bowel tissue.

The authors are to be congratulated on following a noteworthy pathway for innovative surgical research, by first pursuing feasibility in an animal model prior to proceeding with surgical interventions in children under approval by their Ethics Committee. Overall I find the concept appealing but remain cautiously skeptical. As indicated by the authors, the suggestion that skin based flaps are less morbid, simpler to construct or superior to the alternatives may turn out to be true, but there is paucity of data to categorically support or disprove this assumption. Being the developers of the procedure, they are in prime position to establish prospective clinical research protocols to help us answer many of these questions. As with many other things in medicine, with experience we may discover that patient selection is likely to play an important role. For example, skin-based conduits may not be best for children who undergo concomitant augmentation cystoplasty or with multiple prior surgical interventions with incisions in areas that may compromise the blood supply of the flaps.

I sincerely look forward to a favorable response from the surgical community and hope that after experience with the RPM technique grows we can enjoy the expansion of our surgical options based on the foundations set by this elegant study.

\section{REFERENCE}

1. Perovic S: Continent urinary diversion using preputial penile or clitoral skin flap. J Urol. 1996; 155: 14026.

\author{
Dr. Armando J. Lorenzo \\ Pediatric Urology \\ Hospital for Sick Children \\ Toronto, ON, Canada \\ E-mail:ajlmd@aol.com
}

